\title{
The Effect of Laser Shots on Morphological and Optical Properties of Copper Oxide NPs Prepared by Nd-Yag Laser of 1064 nm Wavelengths in Distilled Water
}

\author{
Mahmood M. Kareem* \\ Physics Department, College of Education, University of Garmian, Kalar, Kurdistan Region, Iraq \\ Received 25 August 2021; revised 11 September 2021; \\ accepted 13 September 2021; available online 01 October 2021 \\ doi:10.24271/psr.33
}

\begin{abstract}
This study examines the synthesis of copper oxide nanoparticles (NPs) by using a Q-Switched Nd-Yag (1064 nm) laser on copper foil immersed in Distilled Water (DW). The solution color has changed to light green refers to the production of copper oxide NPs. The generated nanoparticles were studied to determine their characteristics as a function of pulse laser shots and the NPs were obtained by taking 500 and 1000 laser pulse shots on $\mathrm{Cu}$ target. Thin films deposited on both (glass and silicon) substrates were characterized by XRay Diffraction (XRD), Field Emission-Scanning Electron Microscopy (FE-SEM), and Atomic Force Microscopy (AFM) techniques. Later, egarding the colloidal nanoparticles, NPs were instantly characterized by UV-vis spectroscopy and examined by TEM microscopy. The production rate of $\mathrm{Cu}$-NPs concentration in the colloidal solution was measured by atomic absorption spectra type (ICP-OES), which increased by increasing the number of laser shots in the liquid volume. The Energy Dispersion Spectroscopy (EDS) analysis resulted in the presence of copper $(\mathrm{Cu})$ and oxygen $(\mathrm{O})$ elements in the film structure with a nearly stoichiometry ratio. The optical energy gap was decreased to $(2.44 \mathrm{eV})$ with increasing the number of laser shots in the colloidal solution.
\end{abstract}

\section{2021 Production by the University of Garmian. This is an open access article under the LICENSE}

https://creativecommons.org/licenses/by-nc/4.0/

Keywords: Copper oxide, Laser ablation, XRD, FE-SEM, TEM, Energy gap.

\section{Introduction}

Nanomaterials represent an important class of materials in the development of novel devices that can be used in various physical, biological, and biomedical applications, consequently. They are objects of active research in various fields of applications ${ }^{[1]}$. Recently, a number of researchers had worked on the evaluation of the biological activities of metal oxide NPs such as copper oxide from which improved biological and photocatalytic activities have been found ${ }^{[2,3]}$. CuO NPs have attracted particular attention among various metal oxides because they are the simplest copper compounds that shows several unique physical properties such as high-temperature superconductivity and electron correlation effect ${ }^{[4]}$. The considerable antimicrobial activities of metal oxide NPs such as $\mathrm{ZnO}, \mathrm{Fe} 2 \mathrm{O} 3, \mathrm{Fe} 3 \mathrm{O} 4, \mathrm{MgO}, \mathrm{CuO}, \mathrm{TiO} 2, \mathrm{SiO} 2$, have been proved at low temperaturse so the transition metal oxide NPs attract great interest to their notable antibacterial properties ${ }^{[5]}$.

* Corresponding author

E-mail address: mahmood.mohammed@ garmian.edu.krd (Instructor).

Peer-reviewed under the responsibility of the University of Garmian.
$\mathrm{CuO}$ NPs showed particular anticancer, antioxidant potency, and drug delivery which make them a promising tool for biomedical applications, in addition NPs prepared by the laser ablation in liquids have been lately used for manifold and inimitable applications for various fields of technology ${ }^{[6]}$. Lasers opened new physical entries into nanomaterial's process and their characterization. Thus, this technique has various benefits with regards to other typical routs including a huge number of obtainable ablation input variables for controlling the size, shape, intrinsic stoichiometry, and the ability to generate nanomaterials with preferred chemical composition and surface free from chemical contaminations ${ }^{[7]}$. Since Pulse Laser Ablation in Liquids (PLAL) is a clean and green approach for the synthesis of metal as well as metal oxide nanostructures, it has become an increasingly popular approach for producing nanoparticles ${ }^{[8]}$. It is a promising technique for the controlled fabrication of nanomaterials via rapid reactive quenching of ablated species at the interface between the plasma and liquid with high-quality nanoparticles free from chemical reagents ${ }^{[9]}$. When the pulse laser beam is focused on the solid target immersed in DW, the laser immediately interacts with the bulk target and evaporates it into hot and dense plasma. The continuation of this process leads to nucleation and growth in the liquid and NPs will release as a 
result ${ }^{[10]}$. Therefore, PLAL process has received much attention as a novel NPs production technique. Regarding copper oxide, its superior nanoparticles system which inquires the size induced structured transmission and phase stability ${ }^{[11]}$. The simplicity of both the experimental setup and the data analysis has allowed the technique to become a widely used method for NPs generation. Copper oxide nanomaterials have attracted considerable attention among all the metal oxides due to their unique properties. In short, PLAL which combines the physical and chemical mechanism has been used as a rapid and clean approach to synthesize copper oxide NPs with different size and shapes from a pure copper foil immersed in DW. The synthesized colloidal NPs have been studied by using UV-visible spectroscopy and TEM image. Moreover, the deposited thin films were characterized by X-Ray Diffraction (XRD), and FE-SEM scanning microscopy. The size of copper oxide NPs was estimated by Scherer equation according to XRD results.

\section{Experimental Procedure}

\section{1 Materials}

Copper $(\mathrm{Cu})$ foil with dimensions of $0.8 \mathrm{cmx} 1.0 \mathrm{~cm} \times 2 \mathrm{~mm}$ and a purity of $99.99 \%$, from (Alfa Aesar) company were used, and normally immersed in the bottom of a glass vessel filled with distilled water up to $8 \mathrm{~mm}$ of height which then irradiated with the focused output of wavelength $1064 \mathrm{~nm}$ from nanosecond pulsed Nd: YAG laser to generate the copper oxide nanoparticles in distilled water.

\section{1. 1 Laser Source System}

Q-Switched ND: YAG laser system model is (Yinh-V18), with pulses of wavelengths 1064nm and $532 \mathrm{~nm}$ (frequency-doubled), and maximum energy per pulse of $1400 \mathrm{~mJ}$ and pulse width of 10 ns. In this study, $2 \mathrm{~Hz}$ repetition rate and effective spot size of 2.6 $\mathrm{mm}$ with laser flounce $(\mathrm{F}=20.22) \mathrm{J} / \mathrm{cm}^{2}$ and wavelength $1064 \mathrm{~nm}$ were focused by a lens of focal length $(\mathrm{F}=100 \mathrm{~mm})$ to achieve high laser flounce used for ablation process in DW.Also, the cooling system is incorporated into the apparatus to prevent the head gun from overheating.

\section{2 Production of Colloidal NPs}

The fabrication of metal oxide NPs by Q-Switched Nd-Yag laser is an effective technique for NPs production. The copper oxide colloidal NPs of green color compared with distilled water, as in fig. (1) have been generated by letting the (500) and (1000) shots laser of energy $620 \mathrm{~mJ}$ to pass over the surface of bulk pure copper foil immersed in DW at the bottom of $5 \mathrm{ml}$ glass vessel.

The color of DW changed into light green by increasing the number of laser pulse to (500) shots, while the color of colloidal liquid turned to dark green with increasing the number of laser pulse up to (1000) shots ndicating the increasing density of copper oxide NPs production in the solution. The rate of $\mathrm{Cu}$ NPs in the liquid was analyzed by atomic absorption spectra, type (ICP-OES, SPECTRO ACROS), for 500 and 1000 laser shots, the values were (21.35 and 31.42) mg/litter respectively. One drop of the colloidal has been dropped at the center of the piece of p-type $\mathrm{Si}(100)$ and left on the hot plate heater of $100^{\circ} \mathrm{C}$ for 5 minutes, then the prepared samples were tested for surface morphology by
FE-SEM microscopy. Also, the same procedure has been applied for the synthesis of the film on a cleaned glass substrate and the films were tested by XRD. The colloidal liquid itself has been tested by TEM for both laser shots to find the shape and size of copper oxide NPs. Furthermore, optical properties have been studied by UV-Vis. 2600 spectroscopy.

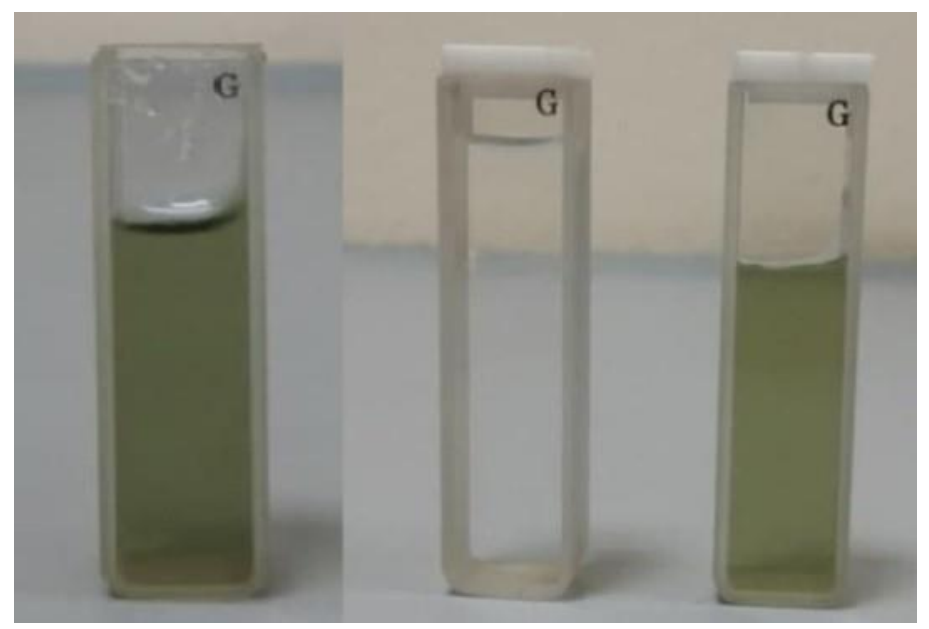

Figure 1: Copper oxide NPs: Left- dark green for (1000) shots Laser: Right -light green (500) shots laser.

\section{Results and Discussion}

\section{Structure Study}

3. 1 XRD Analysis for Thin Films Deposited on both Glass and Silicon Substrates

Copper oxide NPs synthesized by 500 laser shots have been dropped on the glass substrate at $100^{\circ} \mathrm{C}$. Fig.(2) shows the XRD pattern of the film at positions of $2 \theta=21.68^{\circ}$ and $23.55^{\circ}$ which do not belong to any copper oxide standard card, while the strongest peak is at $2 \theta=43.77^{\circ}$, a broadening peak to be contributed to $\mathrm{Cu}$ (111) crystallography orientation and belongs to the reference code No.(00-001-1241). The value of full width at half maximum(FWHM) was 0.52 and by using Scherer's formula ${ }^{[12]}$ the result of crystal size was $16.47 \mathrm{~nm}$ which refers to the poor crystaline film, due to low-temperature of film deposition.



Figure 2: XRD pattern for $\mathrm{Cu}(111)$ thin film on glass substrate at 100 ${ }^{\circ} \mathrm{C}$.

When the film is synthesized by a drop of copper oxide colloidal NPs on p-type Si (100) at $100 \mathrm{oC}$, XRD pattern fig.(3) shows three diffraction peaks at $2 \theta=25.220$ which belong to $\mathrm{Cu} 2 \mathrm{O}$ thin film according to the standard reference code (00-044-0706). 


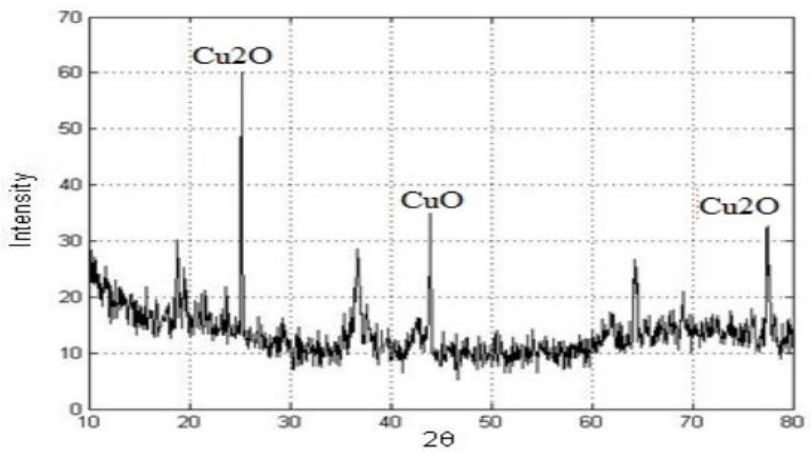

Figure 3: XRD pattern of $\mathrm{CuO}-\mathrm{Cu} 2 \mathrm{O}$ thin film on $\mathrm{Si}(100)$ substrate.

The FWHM value for the peak is 0.1150 which indicates a good crystal grain size of $70.84 \mathrm{~nm}$ and agrees with previous studies ${ }^{[13]}$. The $\mathrm{Si}(100)$ also shows a similar XRD pattern to that grown on glass with a negligible difference $0.18 \mathrm{o}$, but with a narrower diffraction peak, at a position of $2 \theta=43.950$, which belongs to the $\mathrm{CuO}$ crystallography according to the reference code (00-0440706). The crystal size was estimated based on FWHM value of 0.117 and from Scherer's formula was 73.28o. This refers to a good crystallinity of the film deposited on Si substrate concerning glass when resulted in less than $17 \mathrm{~nm}$ for all grown peaks. The third prominent peak on $\mathrm{Si}$ substrate, at the position of $2 \theta=$ 77.39 o belongs to $\mathrm{Cu} 2 \mathrm{O}$ (222) thin film according to the reference code (01-078-2076) and ICSD collection code (063281), with cubic structure and lattice constant of $\mathrm{a}=4.267 \AA$ which is connected with space group pn-3m. The results of thin films on p-type $\mathrm{Si}$ (100) substrate confirm the presence of both phases of copper oxide $\mathrm{CuO}$ and $\mathrm{Cu} 2 \mathrm{O}$, which refer to the presence of multi oxidation states.

While thin film deposited on glass substrate obtained under (1000) shots pulse laser and wavelength $1064 \mathrm{~nm}$, fig.(4) shows pure polycrystalline $\mathrm{CuO}$ phase at angles $2 \theta=35.328 \mathrm{o}$ and $38.541 \mathrm{o}$ in the plane directions (002) and (111) respectively, according to ICSD collection code (067850). As a result, the effect of the number of shots is nearly the same for crystalline growth on glass, while the effect of laser shots is very clear which confirms the presence of $\mathrm{CuO}$ phase structure with respect to the result of (500) shots pulse laser. The average crystalline size calculated was Dave. $=13.048 \mathrm{~nm}$, according to Scherer's formula based on XRD pattern ${ }^{[14-16]}$.

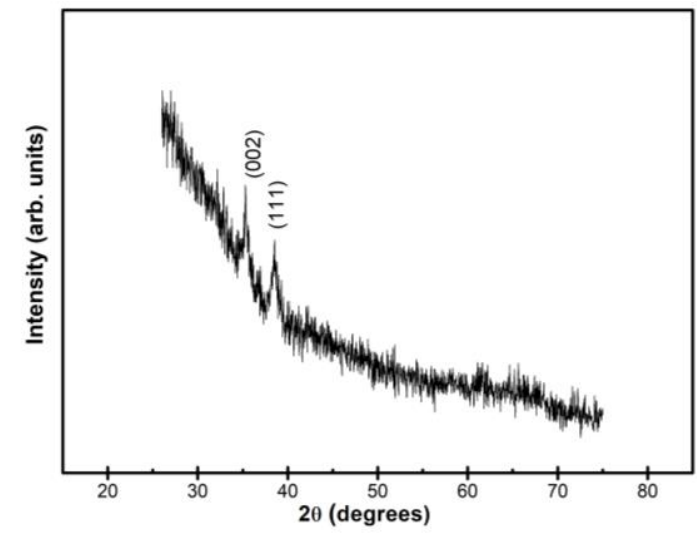

Figure 4: XRD pattern of $\mathrm{CuO}$ NPs prepared on glass substrate at 100 OC by (1000) shots of pulse laser.
$D_{h k l}=\frac{0.89 \lambda}{\beta \cos \theta}$

Where Dhkl is the crystallite size referred to (hkl) peak occurring at a diffraction angle $\theta, \beta$ is the full width at half-maxima of the (hkl) diffraction peak, $\lambda$ is the wavelength of the X-ray used for the diffraction.

\section{2 TEM Image for Synthesized Colloidal Nanoparticles in DW}

TEM was used for high resolution images as well as further analysis of the structure of copper oxide colloidal NPs growth, which were prepared in distilled water by pulse Nd-Yag laser ablation wavelengths of $1064 \mathrm{~nm}$ and 500 laser shots. Figure 5(a) shows small agglomerated nanoparticles with hemispherical shapes and approximately in the range of $20 \mathrm{~nm}$ to $40 \mathrm{~nm}$. The appearance of agglomerated NPs is due to the aging and absence of anti-agglomeration factor in the colloidal aqueous solution since metal nanoparticles tend to agglomerate. On the other hand, TEM analysis by applying image $\mathrm{j}$ on figure 5(b) at a scale bar of $40 \mathrm{~nm}$, which ablated under (1000) laser shots, shows single NPs possess spherical shapes with smaller mean particles size of about $15 \mathrm{~nm}$. The results show that (1000) shots pulse laser represents better ablation condition.

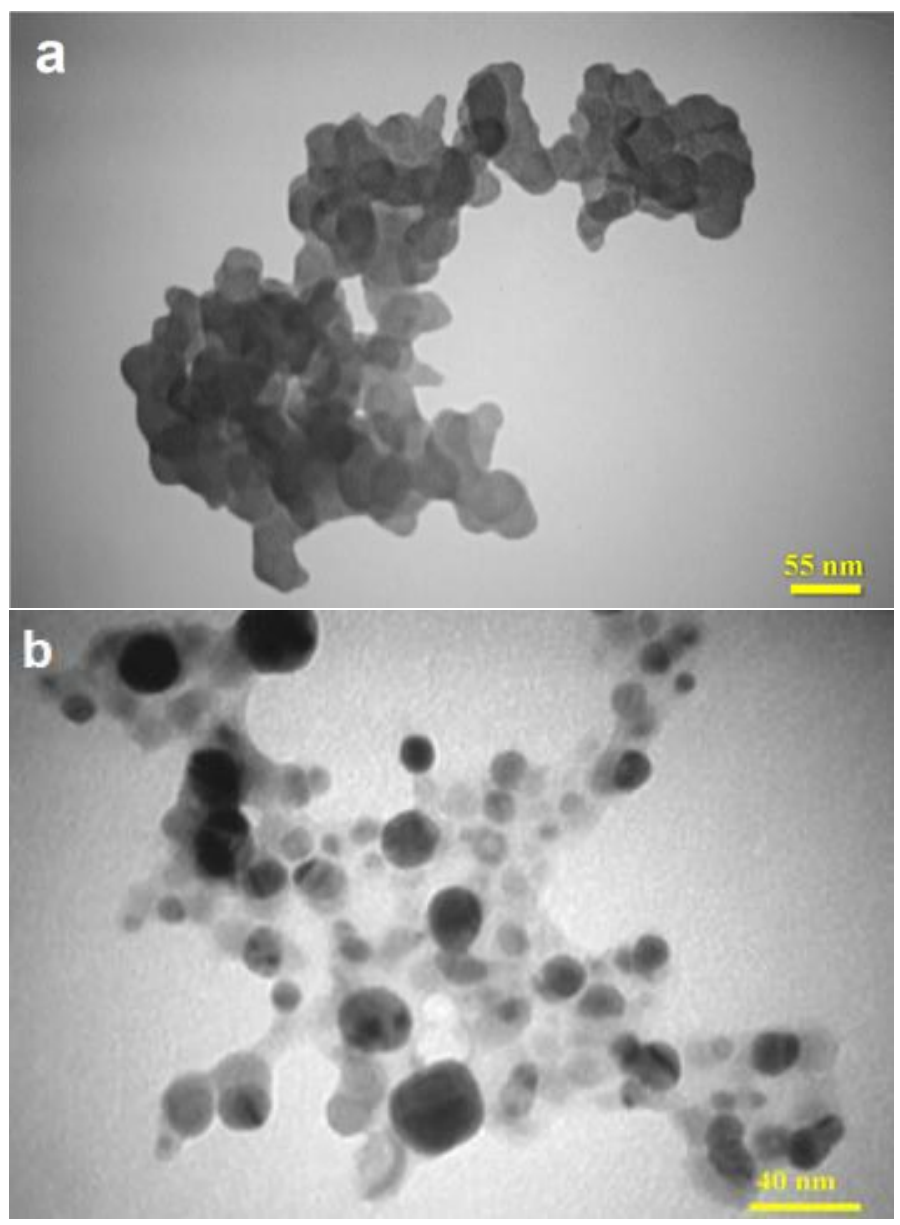

Figure 5: TEM images of copper oxide NPs synthesized (a) by 500 shots, (b) by 1000 shots laser in DW.

Figure (6) displays TEM analysis for the size distribution of copper oxide colloidal NPs. The corresponding size distribution 
of the NPs was produced in DW by 500 laser shots and energy of $620 \mathrm{~mJ}$ per laser pulse, the calculated diameters of the NPs were in the range of $(10-40) \mathrm{nm}$. The result is compatible to a good approach with size of copper oxide NPs calculated by image -J program from the same TEM image which has mean size NPs of about $35.38 \mathrm{~nm}$.

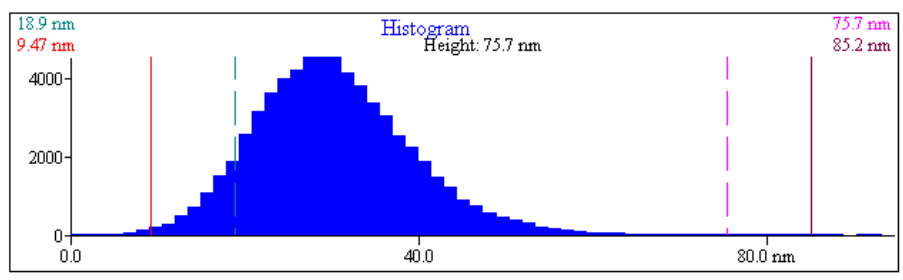

Figure 6: Size distribution of colloidal copper oxide NPs in distilled water.

TEM images of the NPs grown by (1000) shots of pulse laser ablation have a corresponding size distribution of colloidal nanoparticles in distilled water as illustrated in the histogram of fig. (7). The average size of these spherical-shaped NPs is determined to be $15 \mathrm{~nm}$. This reduction in the NPs size with increasing the number of shots which means elongated ablation time is attributed to the extended interaction of NPs with laser light, where higher energy absorption supports the fragmentation of bigger NPs into smaller ones ${ }^{[17]}$.

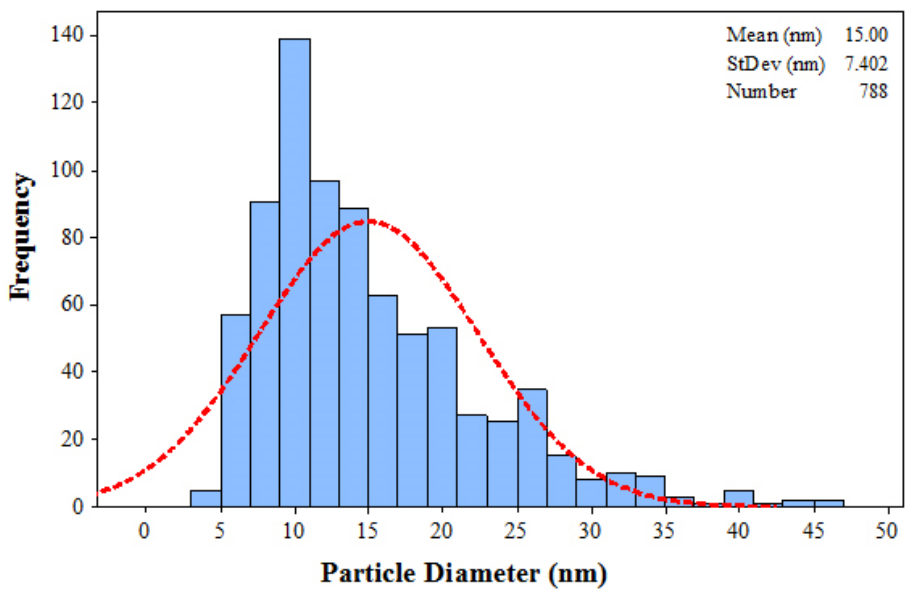

Figure 7: Size distribution of colloidal copper oxide NPs synthesized by (1000) shots laser in distilled water.

\section{Morphology Study and Effects of Laser Shots}

\section{1 (500) Shots of Pulse Laser in DW}

Copper oxide NPs have been dropped on both glass and p-type Si (100) substrates at $100^{\circ} \mathrm{C}$ and synthesized by shooting the pure copper target in DW by (500) pulse of Nd-Yag laser with energy of $620 \mathrm{mj}$ in distilled water. The composed film was studied by FE-SEM. The surface morphology of the sample in figure 8 (a) with $2 \mu \mathrm{m}$ scale bar shows paltry and continuous film distributions with indistinctive morphology on the glass surface and some small particles embedded on the surface. A few rounded particles ranged between 50 to $300 \mathrm{~nm}$ in size can be distinguished and this is maybe due to insufficient substrate temperature for film deposition. In Figure 8 (b), however, at 200 $\mathrm{nm}$ scale, a surface with various nanoparticles ranged approximately from $25 \mathrm{~nm}$ to $150 \mathrm{~nm}$ in size, but most of the particles happen to be identical in shape.
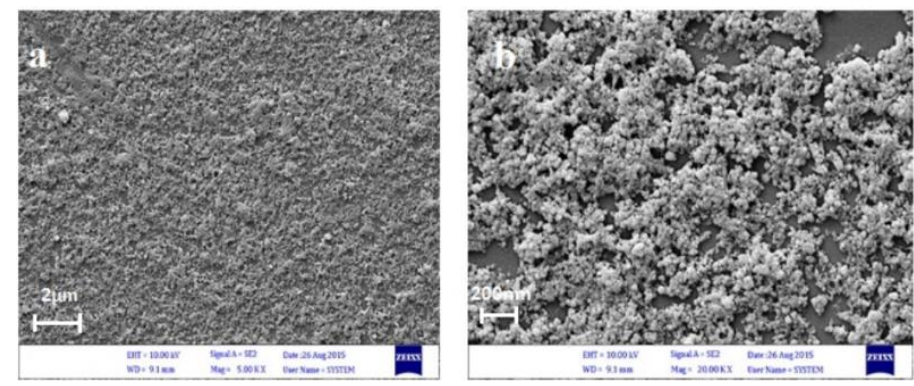

Figure 8: FE-SEM image of copper oxide nanostructure on glass substrate (a) at $2 \mu \mathrm{m}$ scale and (b) on Si (100) substrate at $200 \mathrm{~nm}$.

Better morphology of deposited film on Si (100) might be because their surface atoms have unsaturated dangling bonds with one electron which cause the surface to be reactive and help the diffusion of $\mathrm{Cu}$ atoms into $\mathrm{Si}$ surface. Also, small agglomerated particles appear to constitute the larger particles in size.

\section{2 AFM Analysis of Thin Film on Si (100) Substrate}

In order to scan the thin film deposited on $\mathrm{Si}$ substrate, the resulting map of surface topography by AFM technique has been done by (Auto probe type, U.S.A with software: nanotech electronica) from Day Petronic company. Figure 9(a) shows the 3 -D, AFM image of (5.0x5.0) $\mu \mathrm{m}$ and surface topography of the copper oxide thin film deposited on p-type Si (100) substrate at $100{ }^{\circ} \mathrm{C}$.

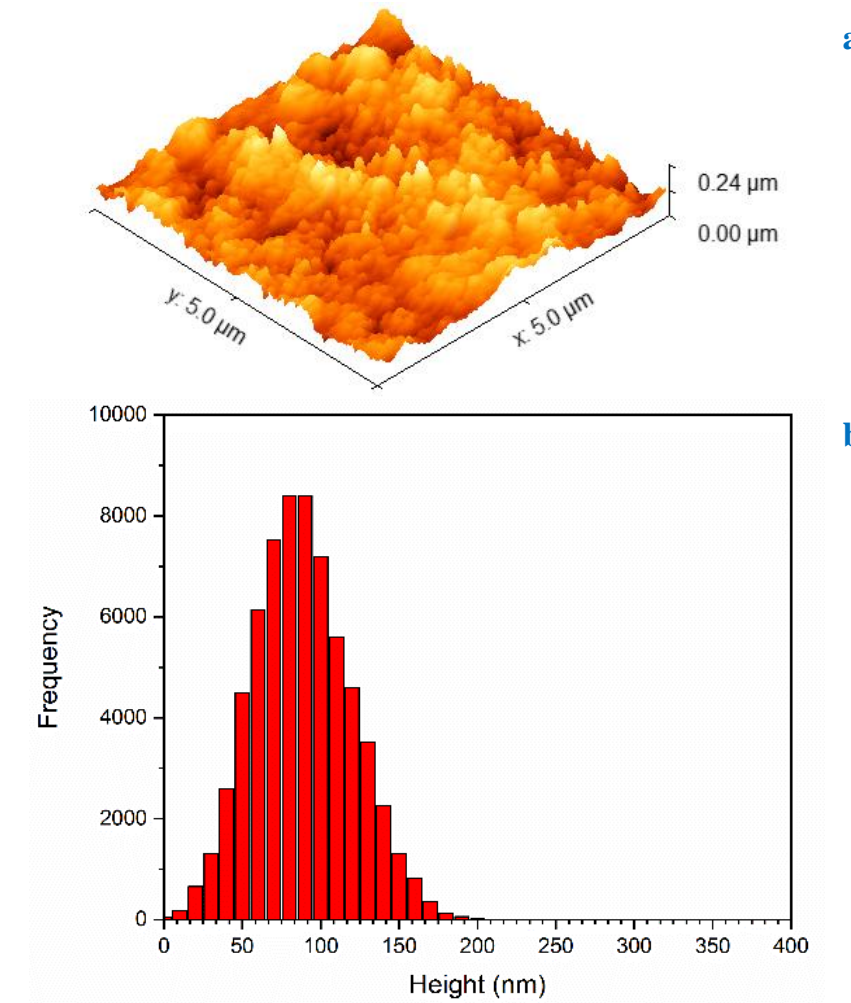

Figure 9: (a) 3-D AFM topographic image $(5.0 \mu \mathrm{m} \times 5.0 \mu \mathrm{m})$ of thin film on $\mathrm{Si}(100)$ (b) particles size distribution.

It is evident that the topography of the film surface has a roughness value Rms of $9.93 \mathrm{~nm}$ and mean height particles of 
$45.57 \mathrm{~nm}$ with some type of nanoparticle accumulation as can be seen from the distribution particles in figure 9(b). The particles ranged from $20 \mathrm{~nm}$ up to $180 \mathrm{~nm}$ in general, but most of the particle diameters distributed frequently in the range $60 \mathrm{~nm}$ to about $140 \mathrm{~nm}$ which is in total agreement with SEM particle size measurements for thin film deposited on Si (100) substrate.

\section{3 (1000) Shots of Pulse Laser in DW}

Copper oxide nanoparticles were synthesized by the same laser wavelengths of $1064 \mathrm{~nm}$ but doubled the number of laser shot pulses (1000) on the surface of pure copper target immersed in DW for the same pulse laser energy of $620 \mathrm{~mJ}$. The colloidal NPs have been deposited on well-cleaned glass substrate at $100{ }^{\circ} \mathrm{C}$ for the sake of comparison with the surface morphology of thin film deposited on glass substrate at (500) laser shots. The SEM image in figure (10) shows perfect and neat copper oxide sphere shapes with diameters ranged approximately between $200 \mathrm{~nm}$ to $500 \mathrm{~nm}$ according to Image-J analysis. The change in morphology was found to be dependent on increasing the number of pulse laser shots up to 1000 pulses to produce copper oxide colloidal NPs. The formation of well-dispersed neat spheres and uniform crystalline structure with different diameters is extremely evident when compared with the films synthesized by 500 shots with the same laser energy on glass substrate which showed aggregated particles with round shapes. Also, the structure shows the aggregation of some nanoparticle materials.

The elemental composition of nanostructure materials for the thin film deposited on glass substrate was investigated and prepared by (1000) pulse laser shots on $\mathrm{Cu}$ target immersed in distilled water. EDS analysis of figure (11) shows the composition of the film which revealed the presence of copper $(\mathrm{Cu}: 76.3)$ wt $\%$ and oxygen element (O: 23.7) wt \% respectively. The results support the atomic ratio distribution of $1: 1$ for $\mathrm{CuO}$ structure if compared with the theoretical calculated value from $\mathrm{CuO}$ molar mass, as shown in table (1).

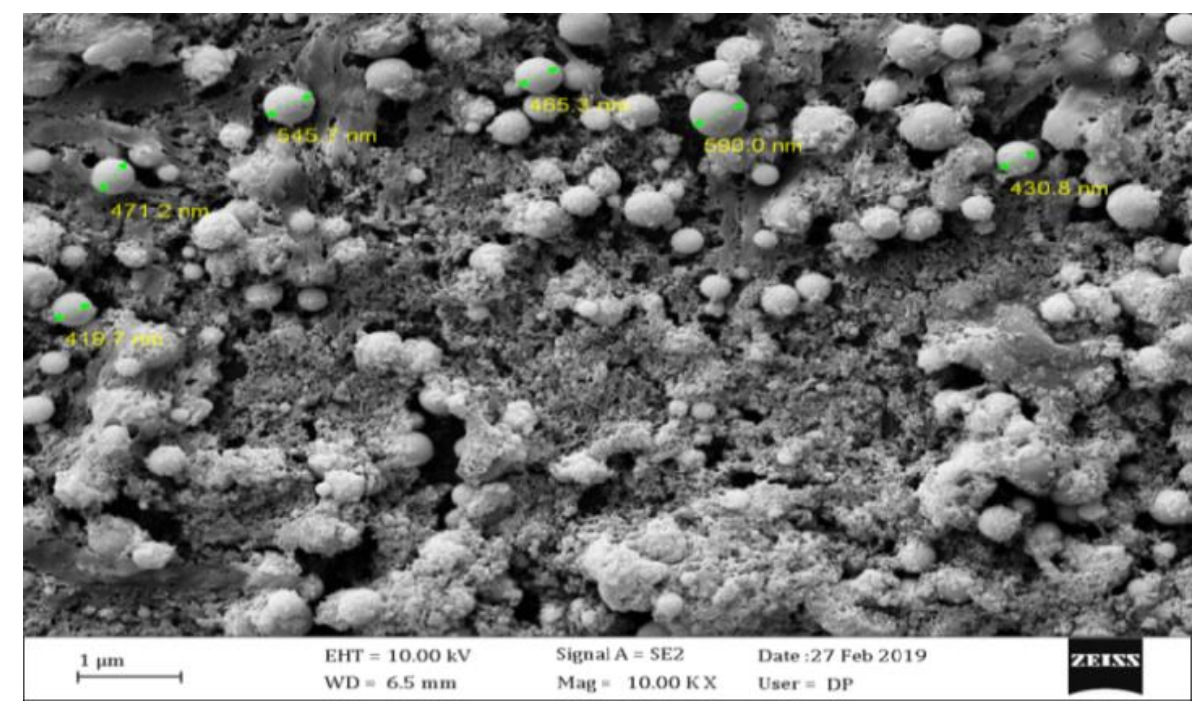

Figure 10: FE-SEM image of copper oxide deposited on glass substrate via (1000) laser shots of energy $620 \mathrm{mj}$.

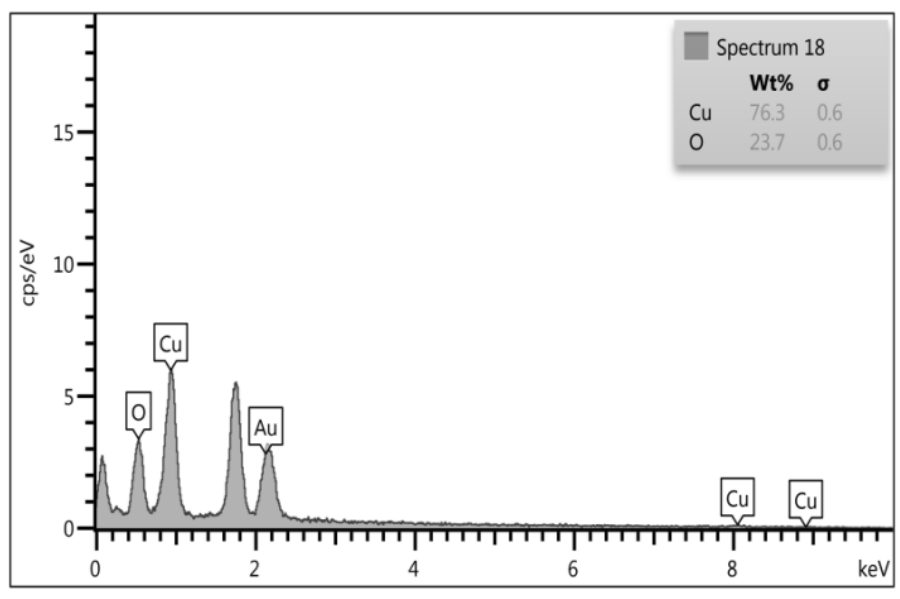

Figure 11: EDS analysis of thin films deposited on glass by (1000) laser shots.

Table 1: Elements weight percent (wt\%).

\begin{tabular}{|l|c|c|}
\hline Elements & Cu wt \% & O wt \% \\
\hline Experimental value & 76.3 & 23.7 \\
\hline Theoretical value & 79.886 & 20.114 \\
\hline
\end{tabular}

\section{Optical Properties}

The synthesis procedure plays a crucial role in controlling the size and shape of the nanostructure and hence detecting different properties of the nanomaterial. Copper oxide colloidal NPs have been prepared by 500 and 1000 pulse of Nd-Yag laser in DW with 1064 wavelengths, which is a novel and easy route for NPs fabrication. The optical properties, absorption, transmission and absorption coefficient have been studied; additionally, the optical energy band gap has been calculated for both numbers of pulses. The thickness of the liquid in the cuvette is supposed to be 1 centimeter.

\section{1 Effect of Laser Shots on Optical Analysis for Colloidal NPs in DW}

\section{1. 2 Absorbance (A)}

Spectroscopic absorption of synthesized colloidal solution of copper oxide NPs in DW is studied in the wavelength range of (200-900 nm) by using UV-VIS spectrophotometer, as shown in figure 12 (a) and (b). The absorption spectra of colloidal NPs display the variation of absorbance as a function of wavelength and from both plots it can be seen that the NPs show higher 
absorption value at shorter wavelength side (UV-region). Fig.12 spectrum(a) done by 500 laser shots shows one shoulder at 270 $\mathrm{nm}$ and the second position is a broadening peak extended from 640 to $720 \mathrm{~nm}$. This may be due to the multi-phase of $\mathrm{Cu}-\mathrm{Cu}_{2} \mathrm{O}-$ $\mathrm{CuO}$ in the colloidal solution. Figure 12(b) which has done by 1000 laser shots, possesses a maximum peak shifted towards a longer wavelength at $305 \mathrm{~nm}$ and terminates with the same behavior as curve (a), but holds an intense peak at $630 \mathrm{~nm}$ showing SPR band of Cu NPs, similar results reported elsewhere ${ }^{[18]}$. The SPR band is affected by particle size and in consequence redshifts it towards a longer wavelength in the visible region. The plot behavior also agrees with literature reports that $\mathrm{Cu}_{2} \mathrm{O}$ strongly absorbs wavelengths below $600 \mathrm{~nm}$. The absorption edge of the colloidal NPs at $360 \mathrm{~nm}$ for figure 12, plot (b) which is toward the shorter wavelength, indicates the higher optical energy band gap and is due to the band edge transition of $\mathrm{Cu}_{2} \mathrm{O}$ [19]. Also, the NPs show the low absorption value (high transmission T, value up to $83 \%$ ) on the higher wavelength side, which is a visible range and higher wavelengths up to $900 \mathrm{~nm}$.

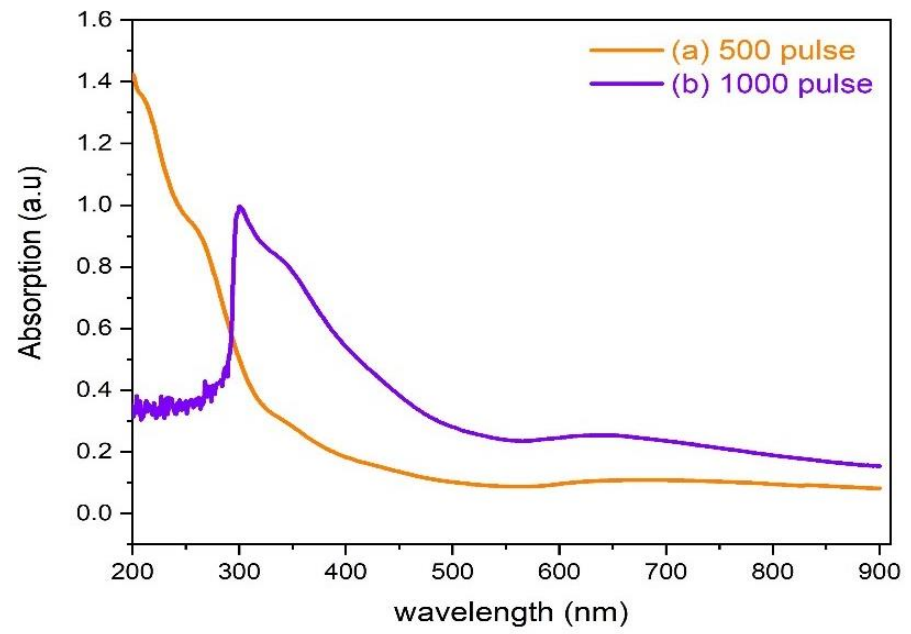

Figure 12: UV-VIS absorption spectra of copper oxide colloidal NPs by (a) 500 shots (b) 1000 shots.

The low absorption phenomenon can be explained by the energy of the incident photon which decreases at the higher wavelengths and cannot interact with atoms of the colloidal media. Thus, the photons will be transmitted instead of absorbing and the interaction of photons and the material with the absorbance of the photons can be observed ${ }^{[20]}$.

\section{1. 3 Optical Energy Band Gap Eg Calculation}

The optical band gab $\mathrm{Eg}$ of $\mathrm{Cu}_{2} \mathrm{O}$ nanoparticles prepared by 500 and 1000 pulses of Nd-Yag laser in DW is calculated from Tauc's relation [21]. The absorption coefficient $(\alpha)$ also has been calculated from the relation below which depends on the absorbance A:

$$
\alpha=2.303 \mathrm{~A} / 2
$$

The optical band gap was carried out by linear extrapolation of the $(\alpha h v)$ versus $(h v)$ curve from the equation

$$
\alpha(\mathrm{v})=\mathrm{A}\left(\mathrm{hv} / 2-\mathrm{E}_{\mathrm{g}}\right)^{\mathrm{m} / 2}
$$

Where $\mathrm{A}$ is a constant, $\alpha$ is the absorption coefficient and $\mathrm{m}$ equals 1 for a direct transition. Figure (13) shows the obtained energy band gap for the $\mathrm{Cu}_{2} \mathrm{O}$ colloidal NPs in case of 500 pulse laser shots which is the direct optical band gap of $2.54 \mathrm{eV}$, similar results have been founded elsewhere ${ }^{[22]}$.

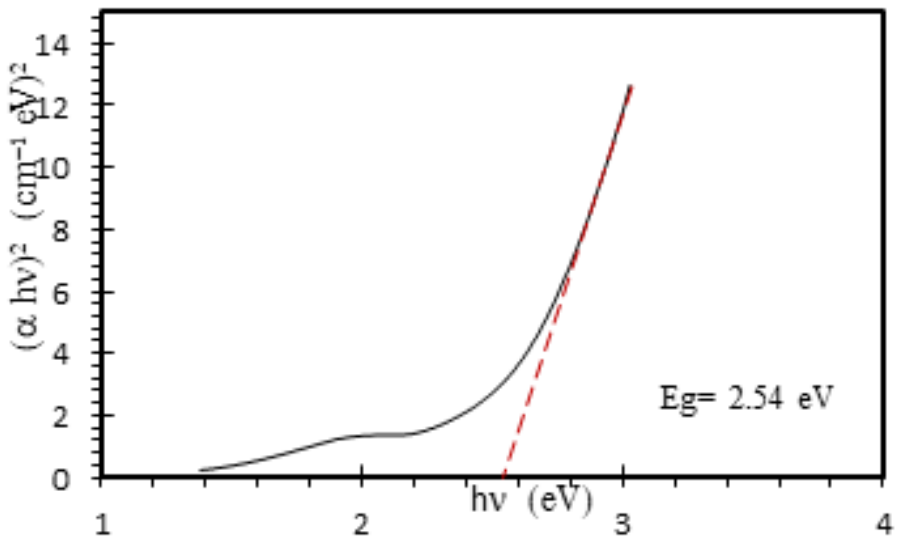

Figure 13: $(\alpha h v) 2$ vs. hv plot for Cu2O colloidal NPs by $1064 \mathrm{~nm}$ laser wavelengths and 500 shots.

In the case of 1000 pulse laser shots, the obtained optical energy gap for the colloidal copper oxide NPs was $2.44 \mathrm{eV}$, as shown in figure (14). It is evident that the results obtained by this method indicate that the $\mathrm{E}_{\mathrm{g}}$ of $\mathrm{Cu} 2 \mathrm{O} \mathrm{NPs}$ is due to the direct allowed transition, and the results of optical band gap are in good agreement with the theoretical value and with previously measured values for $\mathrm{Cu}_{2} \mathrm{O}$ band gap in the literature studies ${ }^{[23,24]}$. The evaluated band gap is a little higher than the direct band gap value of bulk $\mathrm{Cu}_{2} \mathrm{O}$ which is $(2.0-2.17 \mathrm{eV})$. The blueshift in the value of direct band gap of the copper oxide is due to the decrease in particle size as a consequence of the appearance of quantum confinement effects ${ }^{[25]}$.

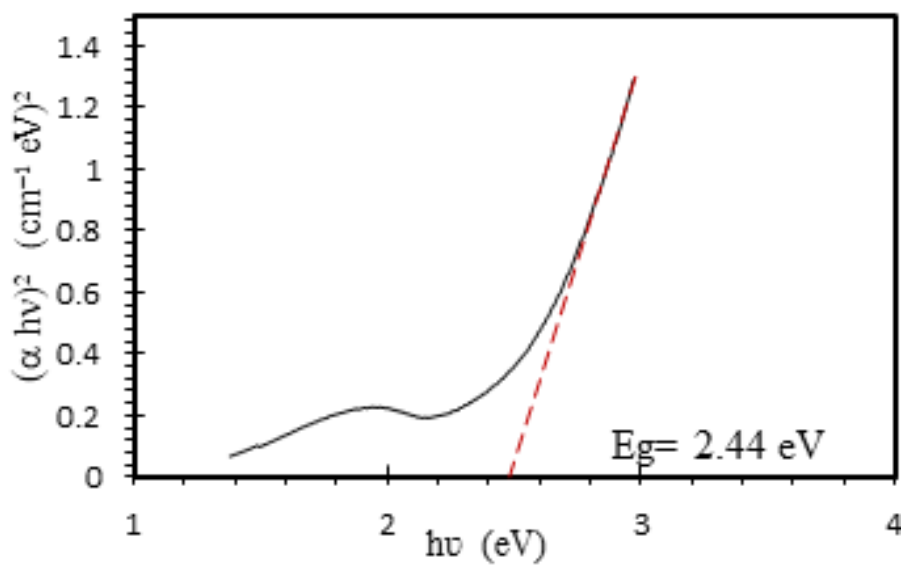

Figure 14: $(\alpha h v) 2$ vs. hv plot for $\mathrm{Cu} 2 \mathrm{O}$ colloidal NPs by1064 nm laser wavelengths and 1000 shots.

\section{Conclusion}

Nd-Yag pulse laser technique on copper metal foil immersed in DW has been successfully used as a clean, safe and one-step physical method to produce the copper oxide colloidal NPs. It is found that the concentration of $\mathrm{Cu}$ NPs in the colloidal liquid increased with increasing the number of laser shots on the $\mathrm{Cu}$ target which produced dark green colloidal. Regarding the deposited copper oxide thin films structure and morphology, it seems to be influenced by the type of substrate (glass or silicon). TEM images of the copper oxide colloidal solution produced by (500) shots of laser show cluster NPs about (20-40) $\mathrm{nm}$ in size with semispherical shapes. However, in the case of (1000) shots, the TEM image and its histogram distribution shows clear change in size and shapes of NPs. The produced NPs were spherical with 
an average size of about (15) $\mathrm{nm}$. The size distribution and agglomeration of NPs were investigated in detail. The shape change of the NPs also has been proved from the FE-SEM images of the morphology of the thin films produced by 1000 pulses. The absorption spectrum of the colloidal NPs produced by 1000 shots results in a prominent SPR peak at a position of $630 \mathrm{~nm}$ which belongs to $\mathrm{Cu}$ metal NPs. The estimated optical band gaps were (2.54 and 2.44) eV. They decreased with increasing the number of laser shots, which most probably indicate the formation of cuprous oxide $\mathrm{Cu}_{2} \mathrm{O}$ NPs. It is evident from the decreasing band gap values that the produced $\mathrm{Cu}$ and $\mathrm{Cu}_{2} \mathrm{O}$ NPs possess bigger sizes of spherical shapes. The absorption spectrum of 1000 laser shots transpires the prominent peak at $305 \mathrm{~nm}$ which belongs to the inter band transition of electrons from valance band of $\mathrm{Cu}_{2} \mathrm{O}$. The results showed the possibility to control the optical energy gap as well as the particle size of the synthesized colloidal NPs by changing the number of laser shots.

\section{References}

1. M. Raffi, F. Hussain, T. Bhatti, J. Akhter, A. Hameed, and M. Hasan, "Antibacterial characterization of silver nanoparticles against E. coli ATCC-15224," Journal of materials science and technology, vol. 24, no. 2, pp. 192-196, 2008.

2. S. Norzaee, B. Djahed, R. Khaksefidi, and F. K. Mostafapour, "Photocatalytic degradation of aniline in water using CuO nanoparticles," Journal of Water Supply: Research and Technology—Aqua, vol. 66, no. 3, pp. 178-185, 2017.

3. M. Aminuzzaman, L. M. Kei, and W. H. Liang, "Green synthesis of copper oxide (CuO) nanoparticles using banana peel extract and their photocatalytic activities." pp. 020016, 2017.

4. A. El-Trass, H. ElShamy, I. El-Mehasseb, and M. El-Kemary, "CuO nanoparticles: synthesis, characterization, optical properties and interaction with amino acids," Applied Surface Science, vol. 258, no. 7, pp. 2997-3001, 2012.

5. R. L. Kalyani, J. Venkatraju, P. Kollu, N. H. Rao, and S. V. N. Pammi, "Low temperature synthesis of various transition metal oxides and their antibacterial activity against multidrug resistance bacterial pathogens," Korean Journal of Chemical Engineering, vol. 32, no. 5, pp. 911-916, 2015.

6. E. Fazio, B. Gökce, A. De Giacomo, M. Meneghetti, G. Compagnini, M. Tommasini, F. Waag, A. Lucotti, C. G. Zanchi, and P. M. Ossi, "Nanoparticles engineering by pulsed laser ablation in liquids: concepts and applications," Nanomaterials, vol. 10, no. 11, pp. 2317, 2020.

7. D. Zeng, K. Yung, and C. Xie, "UVNd: YAG laser ablation of copper: chemical states in both crater and halo studied by XPS," Applied surface science, vol. 217, no. 1-4, pp. 170-180, 2003.

8. J.-P. Sylvestre, A. V. Kabashin, E. Sacher, M. Meunier, and J. H. Luong, "Stabilization and size control of gold nanoparticles during laser ablation in aqueous cyclodextrins," Journal of the American Chemical Society, vol. 126, no. 23, pp. 7176-7177, 2004.

9. A. Fojtik, and A. Henglein, "Laser ablation of films and suspended particles in a solvent: formation of cluster and colloid solutions," Berichte der Bunsen-Gesellschaft, vol. 97, no. 2, pp. 252-254, 1993

10. J. Lam, "Pulsed Laser Ablation in Liquid: towards the comprehension of the growth processes," 2015.

11. K. H. Wong, K. Ananthanarayanan, S. R. Gajjela, and P. Balaya, "Solid state dyesensitized solar cell with $\mathrm{TiO} / \mathrm{NiO}$ heterojunction: effect of particle size and layer thickness on photovoltaic performance," Materials Chemistry and Physics, vol. 125, no. 3, pp. 553-557, 2011.

12. M. Kareem, Z. Khodair, and F. Mohammed, "Effect of annealing temperature on Structural, morphological and optical properties of $\mathrm{ZnO}$ nanorod thin films prepared by hydrothermal method," Journal of Ovonic Research Vol, vol. 16, no. 1, pp. 53-61, 2020.
13. P. Ooi, C. Ching, M. Ahmad, S. Ng, M. Abdullah, H. HASSAN, and Z. Hassan, "Characterizations of cupric oxide thin films on glass and silicon substrates by radio frequency magnetron sputtering,” Sains Malaysiana, vol. 43, no. 4, pp. 617-621, 2014.

14. P. K. Samanta, and P. R. Chaudhuri, "Growth and optical properties of chemically grown $\mathrm{ZnO}$ nanobelts," Science of Advanced Materials, vol. 3, no. 1, pp. 107-112, 2011.

15. A. Mohammad, S. ALIRIDHA, and T. Mubarak, "STRUCTURAL AND MAGNETIC PROPERTIES OF Mg-Co FERRITE NANOPARTICLES,” Digest Journal of Nanomaterials \& Biostructures (DJNB), vol. 13, no. 3, 2018.

16. A. M. Mohammad, "Synthesis and Study the Structural and Magnetic Properties of Cobalt Substituted Strontium Hexaferrite," International Journal of Nanoelectronics \& Materials, vol. 13, no. 2, 2020.

17. B. E. B. Al-Jumaili, Z. A. Talib, A. Zakaria, A. Ramizy, N. M. Ahmed, S. B. Paiman, J. L. Ying, I. B. Muhd, and H. Baqiah, "Impact of ablation time on $\mathrm{Cu}$ oxide nanoparticle green synthesis via pulsed laser ablation in liquid media," Applied Physics A, vol. 124, no. 9, pp. 577, 2018.

18. P. Liu, H. Wang, X. Li, M. Rui, and H. Zeng, "Localized surface plasmon resonance of Cu nanoparticles by laser ablation in liquid media," Rsc Advances, vol. 5, no. 97, pp. 79738-79745, 2015.

19. M. Yin, C.-K. Wu, Y. Lou, C. Burda, J. T. Koberstein, Y. Zhu, and S. O'Brien, "Copper oxide nanocrystals," Journal of the American Chemical Society, vol. 127, no. 26, pp. 9506-9511, 2005.

20. J.-H. Lee, J.-S. Yi, K.-J. Yang, J.-H. Park, and R.-D. Oh, "Electrical and optical properties of boron doped CdS thin films prepared by chemical bath deposition," Thin Solid Films, vol. 431, pp. 344-348, 2003.

21. A. Chen, G. Yang, H. Long, F. Li, Y. Li, and P. Lu, "Nonlinear optical properties of laser deposited CuO thin films," Thin Solid Films, vol. 517, no. 15, pp. 4277-4280, 2009.

22. M. Yang, and J.-J. Zhu, "Spherical hollow assembly composed of $\mathrm{Cu} 2 \mathrm{O}$ nanoparticles," Journal of crystal growth, vol. 256, no. 1-2, pp. 134-138, 2003.

23. J. Pierson, A. Thobor-Keck, and A. Billard, "Cuprite, paramelaconite and tenorite films deposited by reactive magnetron sputtering," Applied surface science, vol. 210, no. 3-4, pp. 359-367, 2003.

24. P. Goel, G. Duragasi, and J. Singh, "Copper (I) oxide micro-cubical structures formation by metal organic chemical vapor deposition from copper (II) acetylacetonate," Journal of Materials Science, vol. 48, no. 14, pp. 4876-4882, 2013.

25. N. Semaltianos, S. Logothetidis, W. Perrie, S. Romani, R. Potter, M. Sharp, P. French, G. Dearden, and K. Watkins, "II-VI semiconductor nanoparticles synthesized by laser ablation," Applied Physics A, vol. 94, no. 3, pp. 641, 2009. 